\title{
Modeling of Surface Roughness during Conventional Turning using a Hybrid GA-ANN Based Model
}

\author{
Ranganath. M. Singari ${ }^{1}$, G. S. Bajwa ${ }^{2}$, Prateek ${ }^{3}$ Praveen $^{4}$, Prateek Kalyani ${ }^{5}$, \\ Shadab Ahmad ${ }^{6}$ \\ 1,3,4,5,6 (Mechanical, Production \& Automobile Eng. Dept., Delhi Technological University, Delhi, India) \\ ${ }^{2}$ (Mechanical Engineering Department, G.N.D. Institute of Technology, Delhi, India)
}

\begin{abstract}
In the present study a hybrid GA-ANN based model has been developed for the prediction of Surface Roughness obtained in a conventional turning process. Surface roughness is a very important property of a machined part and is hard to predict due to the complex nature of turning process. Artificial neural networks are intelligent tools, capable of modeling complex non-linear relationships by simulating the working process of a nervous system. Genetic Algorithm is a powerful and efficient global optimization method. These two tools are coupled in the present study to accurately model turning process. A significant improvement in predicted values is observed in comparison to a simple ANN model.
\end{abstract}

Keywords: Artificial Neural Networks, Genetic Algorithms, MATLAB, Surface Roughness, Turning.

\section{Introduction}

Conventional turning process is one of the most important metal cutting process used in the manufacturing industry. In the turning process a workpiece is rotated against a tool which results in removal of metal from the outer surface of the workpiece and production of a cylindrical job. The current Economic requirement is the production of high quality products with lower tolerances and improved surface finish along with simultaneous minimization of the machining time and the total manufacturing cost. Surface roughness is a very important property of a machined part and is responsible for its various functional attributes such as appearance, fatigue resistance, friction coefficient, lubricant holding capacity and stress concentration etc. The surface finish of a turned part depends on various factors such as depth of cut, cutting speed, nose radius, cutting forces, tool wear and vibrations. All these various parameters need to be controlled in order to achieve the desired surface finish during the turning process. In the present study a hybrid GA-ANN model is presented which can be used to predict the surface roughness.

\section{Artificial Neural Network (ANN)}

An artificial neural network is a machine learning method developed by Warren McCulloh and Walter Pitts in 1943. ANN is capable of modeling complex non-linear relationships by simulating the working process of a human brain.

Despite the fact that the neurons present inside the brain are very slow in comparison to the electronic logic gates used in computers. Neurons require few milliseconds to respond to a stimulus while logic gates can process data within nanoseconds, yet the brain is able to solve many complex problems, more efficiently than a computer for example recognizing a face, due to its massively complex network of neuron. Billions of neurons are present inside a human brain and each neuron is connected to millions of other neurons via the synapse. These neurons exchange electrochemical signals with each other, with different synaptic strengths. Each neuron is capable of responding to an input signal. When the total signal received by a neuron exceeds the threshold value, the neuron fires and sends signal to the neighboring neurons. This process is said to be the basis of memory [11].

ANN also has a network of interconnected neurons, which are arranged in the form of layers. This arrangement and interconnection of neurons constitutes the network topology. A feed forward back- propagation network with one hidden layer which is drawn with the help of solidworks is shown in fig. 1. 


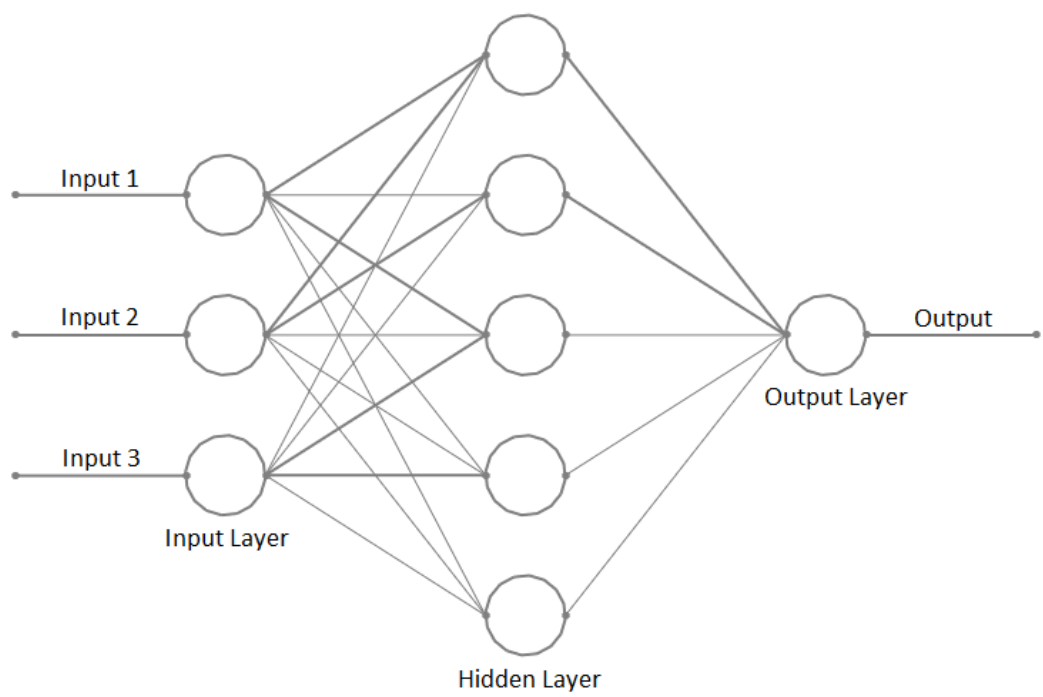

Fig. 1: Feed forward back- propagation network with one hidden layer

These artificial neurons functions like a real neuron. Each neuron-neuron connection has some weight associated with it, and an activation function which fires these neurons, when threshold value is reached. Common activation functions are tan-sigmoid and log-sigmoid functions.

A learning algorithm adapts the weights and bias values of a network to implement desired behavior. Levenberg-Marquardt back propagation algorithm is a commonly used learning algorithm.

The back-propagation algorithm used in supervised training of a neural network has a disadvantage that it is based on gradient descent methods, i.e. these algorithms move from one solution to another along the path having the steepest descent, and hence they easily get trapped in a local optimum. These local optimum solutions have low generalization, i.e. they adequately fit on the training data points, but the error is high on the out of data samples [8]. Hence a neural network needs to be trained a number of times by randomly initializing the weights and bias of the network. Although this approach also does not guarantee giving the optimum solution in case of problems having multiple local optima. A global search algorithm such as the Genetic Algorithms, which do not work on a gradient method, acts as a powerful and efficient tool for finding the optimum weight and bias configuration of a neural network that has well off sample data performance [4].

\section{Genetic Algorithms (GA)}

Genetic Algorithms are evolutionary computation techniques that are based on the "Darwin's theory of Natural Selection". This technique of mimicking nature's selection process in a computer program for solving optimization problems was first introduced in 1975 by John Holland in his book "Adaption in natural and Artificial Systems". Genetic algorithms are a stochastic computer program that works with a population of competent solutions and iteratively improves the population performance in terms of a suitable fitness function, thus finding an optimal solution after several generations/iterations. The fact that GA works with a set of possible solutions (population) instead of a single solution makes it less prone to getting trapped in a local optimum. The non-requirement of information related to gradient of the solution also gives GA an inherent advantage over methods based on gradient methods such as steepest descent method and stochastic hill climbing method.

In the genetic algorithms an initial population is seeded to the program which is usually chosen randomly to induce diversity in the population. Each solution is encoded in the form of a chromosome, usually binary encoding is used for representing the chromosomes and i.e. each gene is represented with either 0 or 1 . But in the present study a real value encoding of the chromosomes is used, due to the large number of variables present, which will make a binary encoding slow and inefficient. Then iteratively a series of genetic operators are applied on this population up to certain generations to obtain a solution with the optimum fitness value. The fitness value is calculated using a suitable fitness function, which defines the superiority of a solution over other. Usually mean square error is used as the fitness function. The various genetic operators are:

1. SELECTION: Pair of solutions, called parents is selected from the population for reproduction on the basis of their fitness value. Solutions with high fitness values have higher chances of selection.

2. CROSSOVER: The parents are then bred using a suitable algorithm, and the off-springs are used to create a new population of possible solutions. 
3. MUTATION: The new population is mutated time to time with a given mutation probability for exploring new areas of research within the search space, and prevents trapping in a local optimum.

Genetic algorithms have a disadvantage that they are unable to efficiently reach the exact global optimum solution once the near optimum solution is reached and the population gets dominated with a best chromosome. After that the convergence rates diminishes very rapidly and relies mainly on the mutations occurring in the population [4]. To overcome this problem a large population size with enough genetic diversity needs to be chosen initially, which increases the computation time enormously.

The main objective of this paper is to generate a hybrid GA-ANN based model for studying the surface roughness during conventional turning. Hence in this study the features of genetic algorithms are exploited only to reach the near optimum weights and bias values, and after that, a feed forward back propagation algorithm is used to reach the optimum value.

\section{Literature Review}

In the past number of attempts have been made by researchers to model the turning process, using empirical and semi-empirical models based on neural networks, example being:

Davim et al [5] used an error back propagation training algorithm [EBPTA], to develop an ANN model of surface roughness obtained on turning of free machining steel using cemented carbide tools. The experiments were designed using Taguchi's $\mathrm{L}_{27}$ orthogonal arrays. He used this model to study the effects of cutting parameters (depth of cut, cutting speed and feed) on the surface roughness obtained.

DurmusKarayel [2], developed an ANN model for the prediction of surface roughness using the Scaled conjugate gradient algorithm (SCGA) training method. He used this model to develop a control system, for a computer numerically controlled (CNC) lathe, which is used to determine the machining parameters for desired surface finish requirement. The control system was tested on a CNC lathe and it was concluded that ANN produced an accurate relationship between the cutting parameters and the surface roughness.

Ranganath M. S. et al studied optimization of surface roughness and material removal rate on conventional dry turning of Aluminium (6061) using Taguchi analysis [16]. Diwakar Reddy. V. et al [6], measured the surface roughness obtained during dry turning of mild steel work piece using an uncoated carbide insert, under different machining conditions. He used these observations to develop a 3 layered feed forward back propagated neural network model for prediction of surface roughness. He compared the performance of neural network models with different number of hidden layers and selected 25 neurons in the hidden layer as the best architecture. Levenberg-Marquardt (LM) algorithm was used to train the network, tan sigmoidal and pure line transfer functions were used in the hidden and output layers respectively.

Ranganath. M. S. et al, "Experimental Investigation of Surface Roughness and Cutting Force on Conventional Dry Turning of Aluminium (6061)" June 2015 [1], has studied the effect of cutting speed, depth of cut feed rate and on surface roughness, in turning of Aluminium (6061). Taguchi's orthogonal arrays were used for the Design of experiments (DOE), to analyze the influence of the turning parameters on the surface roughness. Analysis of Variance (ANOVA) technique was then used for optimization of the surface finish. It was concluded that the minimum surface roughness is obtained when the feed and depth of cut are set at the lowest possible values. Ranganath M. S. et al studied the analysis of surface roughness in turning process using ANN $[9,12,13,14,15]$ and found that ANN models can be utilized in turning process.

In the past few years a recent surge is seen among researchers to combine the abilities of neural networks to model complex non-linear relationships with the global search capability of a Genetic Algorithm. The genetic algorithms can be coupled with ANN in a number of ways, they can be used to optimize the network topology and/or they can be used as an alternative to the standard training algorithms, to find the optimum weight and bias values for each connection [8]. Genetic algorithms are also used to optimize the machining parameters, using a trained neural network to calculate the fitness function. These approaches have been applied by many researchers for modeling various manufacturing processes.

Venkatesan et. al. "A genetic algorithm-based artificial neural network model for the optimization of machining processes, 2005" [7] made a GA based ANN model of the turning process, in which he used the genetic algorithm to find out the optimal connection weights, and compared it with a simple back propagation network model. He used a 5 - 5 - 4 neural network architecture and a real value encoding with 225-digit long chromosome, which represented the total 45 connection weights present in the architecture. For the GA he used a 225 population size with rank selection and two point's crossover method. By comparing the two methods he found only a nominal saving of computing time with the GA-ANN approach.

G. Krishna Mohan Rao et al [10], used a hybrid GA-ANN based approach to model surface roughness during EDM process, in which GA were used to optimize the weight factors of the network. The GA based 
hybrid approach reduced the production data errors from a maximum value of $5.68 \%$ in case of neural network model to $1.8 \%$ using the hybrid approach.

\section{Network Modeling}

In the present study experimental results obtained in the paper Ranganath. M. S. et al, "Experimental Investigation of Surface Roughness and Cutting Force on Conventional Dry Turning of Aluminium (6061)" June 2015 [1], given in Table 1, is used to develop the hybrid GA based neural network model (GA-ANN).

Table 1. Experimental results

\begin{tabular}{|l|l|l|l|}
\hline DoC & SPEED & FEED & ROUGHNESS \\
\hline 0.6 & 156 & 0.05 & 2.2 \\
\hline 0.6 & 289 & 0.1 & 3.21 \\
\hline 0.6 & 409 & 0.15 & 4.25 \\
\hline 1.2 & 156 & 0.05 & 1.77 \\
\hline 1.2 & 289 & 0.1 & 2.46 \\
\hline 1.2 & 409 & 0.15 & 3.99 \\
\hline 1.8 & 156 & 0.05 & 2.53 \\
\hline 1.8 & 289 & 0.1 & 2.95 \\
\hline 1.8 & 409 & 0.15 & 3.64 \\
\hline
\end{tabular}

For the network modeling, depth of cut (DoC), cutting speed and feed rate are selected as the input variables and surface roughness is taken as the target values. Hence a model with 3 input neurons and 1 output neuron is made.

A custom program developed by the authors in MATLAB software, is used to compute the optimum parameters of the neural network model. This program uses a GA to select the number of neurons in the hidden layer, training algorithm and the transfer functions of the hidden and output layers. The program works by selecting the architecture that best fits the experimental data. The network parameter settings given by the program is shown in table 2. An ANN is then trained to predict surface roughness using these parameters.

Table 2. ANN Parameter Settings

\begin{tabular}{|l|l|}
\hline Network Type & Feed forward back propagation \\
\hline Training function & Levenberg Marquardt (LM) \\
\hline Learning function & LEARNGDM \\
\hline Performance function & MSE \\
\hline Transfer function of hidden layer & TANSIG \\
\hline Transfer function of output layer & PURELIN \\
\hline Number of hidden layers & 1 \\
\hline Number of neurons in Hidden layer & 12 \\
\hline
\end{tabular}

Then another custom GA based program runs a genetic algorithm that selects the near optimum weight and bias values of the neural network. The parameter settings used for the genetic algorithm program is shown in Table 3. Due to the stochastic nature of genetic algorithms, the program needs to be run a number of times. The weights and bias values of the best result given by GA are then used to train another neural network initialized using these weights and bias values.

Table 3. Parameter Settings of GA

\begin{tabular}{|l|l|}
\hline Population Size & 200 \\
\hline Population Type & Double Vector \\
\hline Selection Function & Stochastic Uniform \\
\hline Crossover Method & Scattered Crossover \\
\hline Mutation Method & Gaussian \\
\hline Mutation Probability & 0.01 \\
\hline Stopping Criterion & Tolerance Value \\
\hline Tolerance Value & $10^{-15}$ \\
\hline
\end{tabular}

\section{Results and Observations}

The network is trained with the architecture selected by the GA and the resulting regression plot is shown in Fig. 2. Weights and biases of this network are then optimized using the genetic algorithm and the regression plots resulting from the network are shown in Fig. 3. Fig. 4 shows the mean and best fitness value plot of the genetic algorithm used to optimize the weights and biases. 

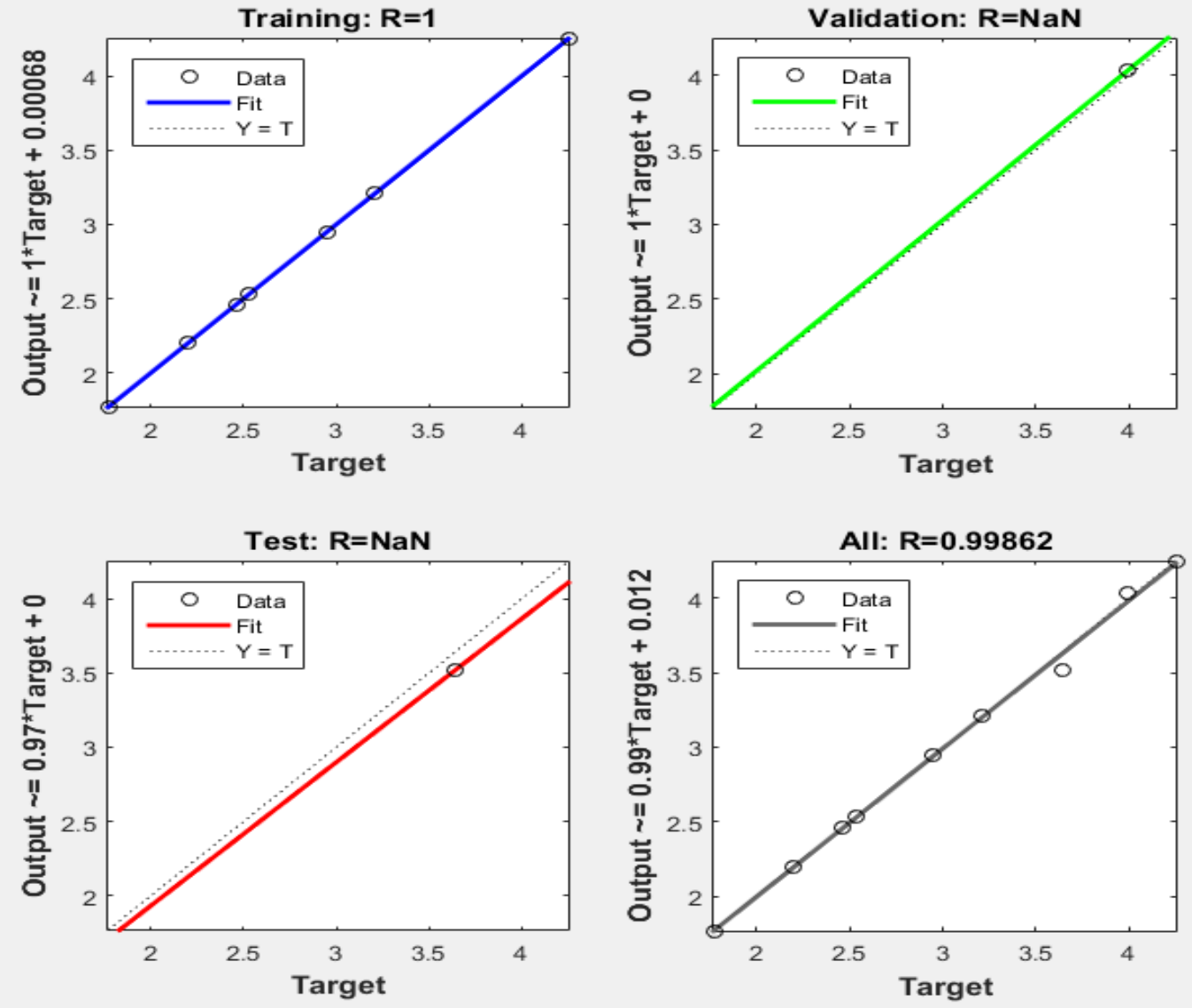

Fig. 2 - Regression plot of ANN model
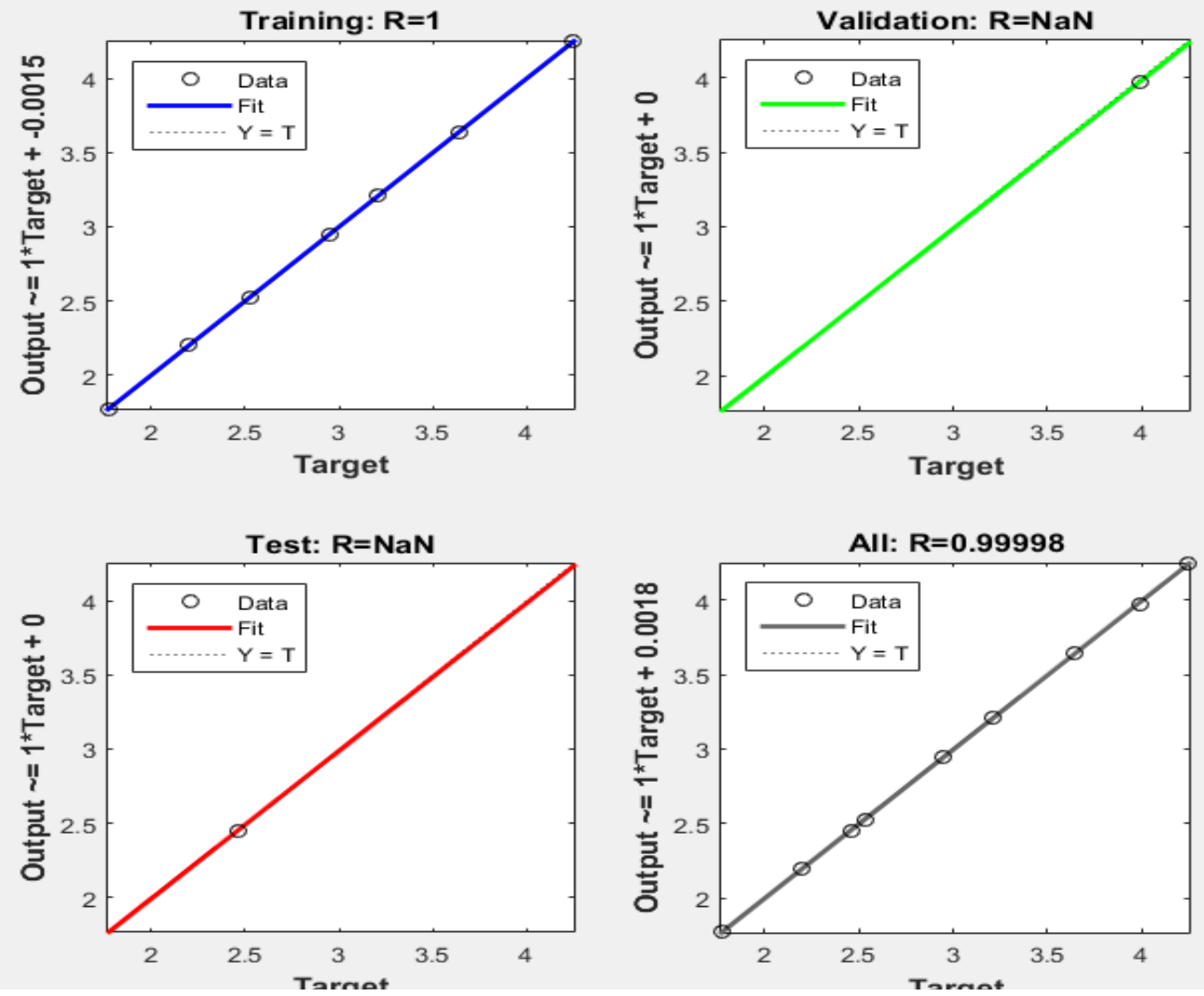

Fig. 3 - Regression plot of Hybrid GA-ANN model 


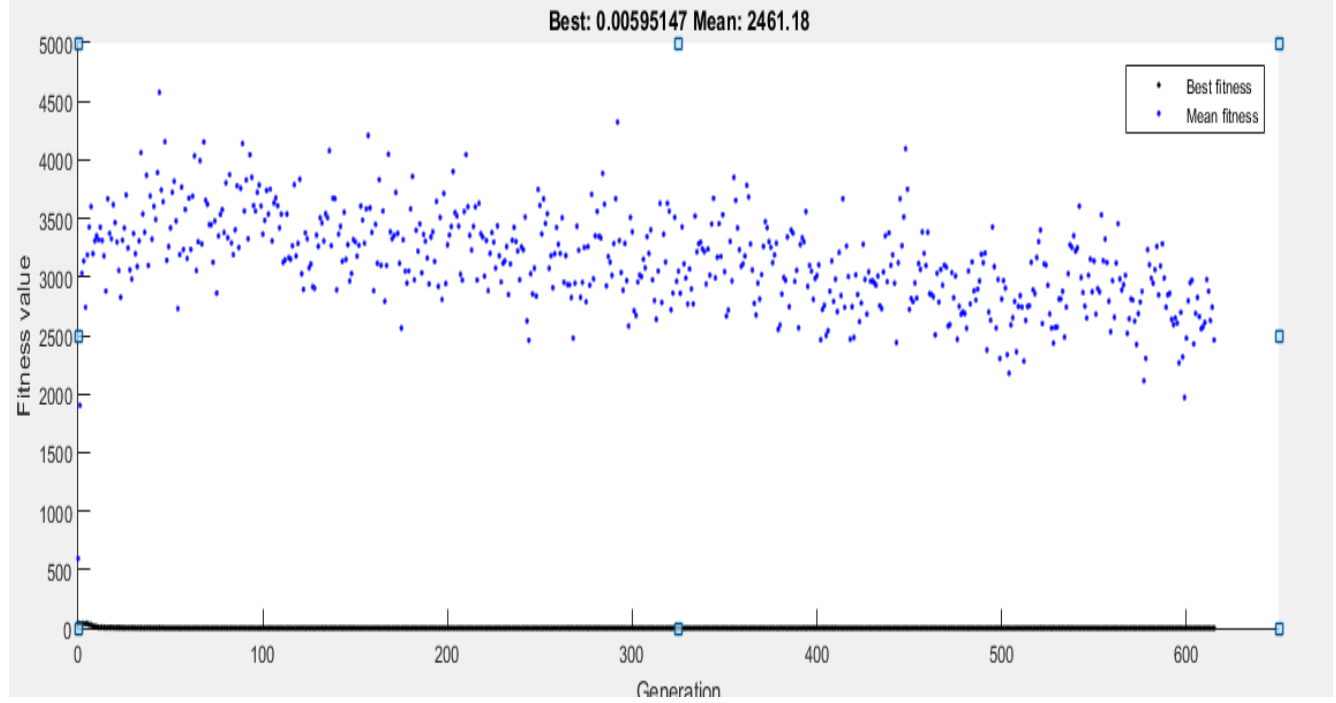

Fig. 4 - Variation of mean and best fitness vs. generation plot

The values predicted by the feed forward (ANN only) network and the hybrid model, along with the percentage errors between the actual and predicted values, is shown in table 4.Fig. 5 and 6, shows the comparison between experimental and the predicted values of Surface Roughness.

Table 4. GA-ANN Predicted Values

\begin{tabular}{|l|l|l|l|l|}
\hline Exp. Value & ANN Predicted Values & \% error & GA-ANN Predicted Values & \% error \\
\hline 2.2 & 2.1998 & 0.0105 & 2.2 & $2 \mathrm{E}-05$ \\
\hline 3.21 & 3.2103 & 0.0099 & 3.21 & $1.11 \mathrm{E}-5$ \\
\hline 4.25 & 4.2500 & 0.0002 & 4.2500 & $2.5 \mathrm{E}-05$ \\
\hline 1.77 & 1.7697 & 0.0149 & 1.7700 & 0.0015 \\
\hline 2.46 & 2.4626 & 0.1057 & 2.4502 & $\mathbf{0 . 3 9 9 6}$ \\
\hline 3.99 & 4.0293 & 0.9846 & 3.9730 & 0.4254 \\
\hline 2.53 & 2.5305 & 0.0202 & 2.5264 & 0.1427 \\
\hline 2.95 & 2.9482 & 0.0618 & 2.9496 & 0.0135 \\
\hline 3.64 & 3.5177 & $\mathbf{3 . 3 5 9 6}$ & 3.6399 & 0.0027 \\
\hline
\end{tabular}

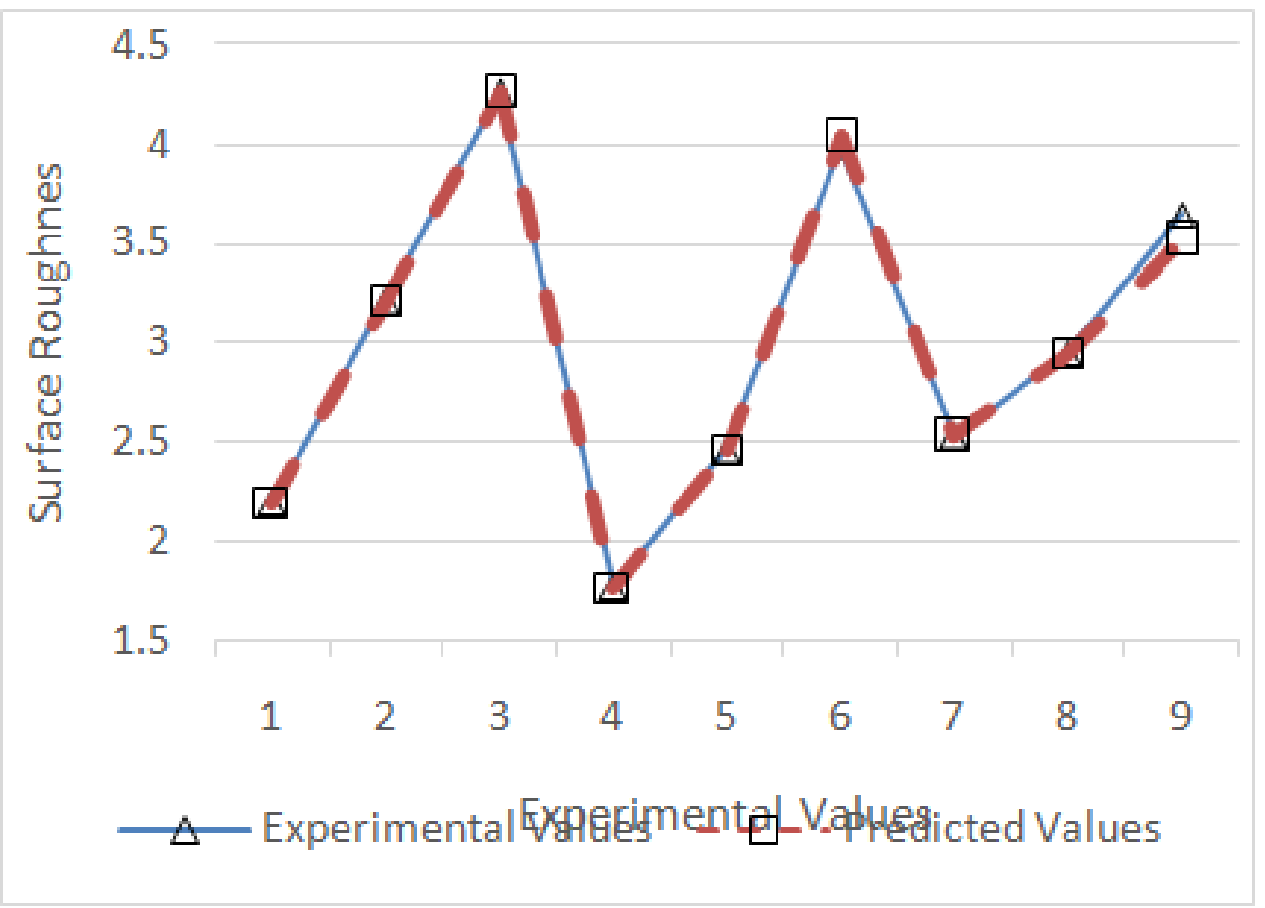

Fig. 5-Comparison between Experimental and Predicted Surface Roughness (ANN) 


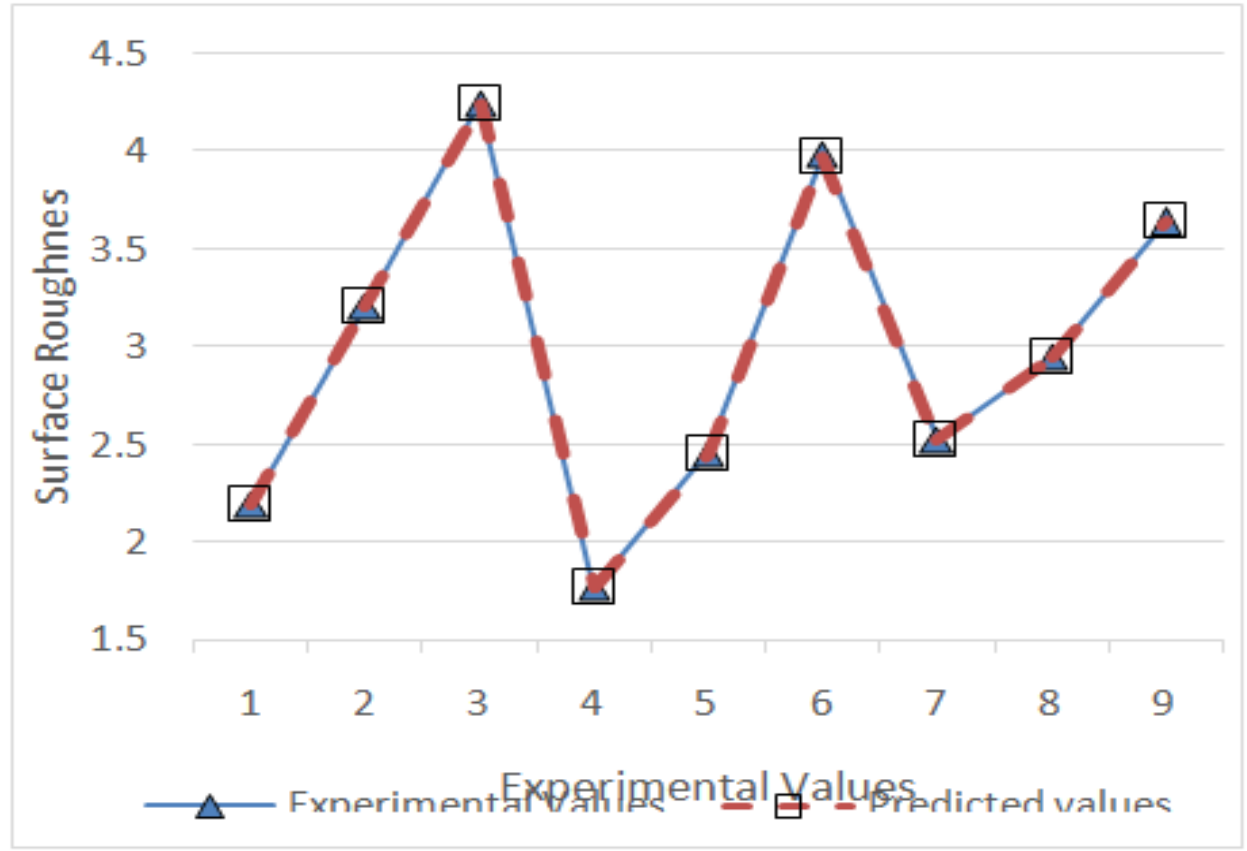

FIg. 6-Comparison between Experimental and Predicted Surface Roughness (GA-ANN)

From the above table it can be seen that the error is significantly reduced when GA is used to calculate the near optimal weights and bias values.

\section{Conclusions}

1. Hybrid GA-ANN model for the surface roughness prediction of Aluminium 6061 material has been developed.

2. Two different approaches for modeling are compared, i.e. using ANN only and a GA-ANN hybrid model.

3. A major reduction in time and effort is observed compared to the trial and error approach because the selection of optimum architecture of the ANN model is done using genetic algorithm.

4. A considerable amount of reduction in percentage error is seen, when network weights and bias values are optimized using Genetic Algorithm.

5. GA-ANN hybrid model performed well, and the errors are within agreeable limits in comparison with ANN model.

\section{References}

[1]. Ranganath M. S., Vipin, R. S. Mishra, Parshvam Jain, Sushil Kumar, "Experimental Investigation of Surface Roughness and Cutting Force on Conventional Dry Turning of Aluminium (6061)" International journal of modern Engineering Research, Vol.5(6), 2015, pp 14-23.

[2]. Durmus Karayel, "Prediction and control of surface roughness in CNC lathe using artificial neural network" Journal of Materials Processing Technology Vol.209(7), 2009, pp 3125-3137

[3]. Ilhan Asiltürk, Mehmet Çunkas, Selçuk, "Modeling and prediction of surface roughness in turning operations using artificial neural network and multiple regression method" Elsevier, Expert Systems with Applications Vol. 38, 2011, pp 5826-5832.

[4]. Hiroaki Kitano, "Empirical Studies on the Speed of Convergence of Neural Network Training using Genetic Algorithms", proceedings of Association for the Advancement of Artificial Intelligence (AAAI), 1990.

[5]. J. Paulo Davim, V. N. Gaitonde, S. R. Karnik, "Investigations into the effect of machining of cutting conditions on surface roughness in turning of free machining steel by ANN models", Journal of Materials Processing Technology, vol. 205, 2008, pp 1623.

[6]. Diwakar Reddy.V, Krishnaiah.G, A. Hemanth Kumar, Sushil Kumar Priya, "ANN based predication of surface roughness in turning", International Conference on Trends in Mechanical and Industrial Engineering (ICTMIE2011) Bangkok Dec., 2011.

[7]. D. Venkatesan, K. Kannan, R. Saravanan, "A genetic algorithm-based artificial neural network model for the optimization of machining processes", Neural Computing and Applications, Vol. 18 (2) 2009, pp 135-140.

[8]. Randall S. Sexton, Robert E. Dorsey, Naheel A. Sikander, "Simultaneous optimization of neural network function and architecture algorithm", Decision Support Systems Vol. 36, 2004, pp 283-296.

[9]. Ranganath. M. S., Vipin, R. S. Mishra, "Application of ANN for Prediction of Surface Roughness in Turning Process: A Review", International Journal of Advance Research and Innovation, Vol. 3, 2013, pp 229-233.

[10]. G. Krishna Mohan Roy,G.Rangajanardhaa, D. Hanumantha Rao, M. Sreenivasa Rao, "Development of hybrid model and optimization of surface roughness in electric discharge machining using artificial neural networks and genetic algorithm", Journal of Materials Processing Technology Vol. 209, 2009, pp 1512-1520.

[11]. Raứl Rojas," Neural Networks a Systematic Introduction”, Springer publications Berlin 1996.

[12]. Ranganath M. Singari, "Analysis of Surface Roughness in Turning Process Using Artificial Neural Network", V ${ }^{\text {th }}$ International Symposium on "Fusion of Science \& Technology", New Delhi, India, January 18-22, 2016, ID: 2016-ISFT-163. 
[13]. Ranganath M S, Vipin, Sudhanshu Maurya, SonuYadav, "Parametric Analysis of Surface Roughness Studies in Turning Using Artificial Neural Network", International Journal of Advance Research and Innovation, Vol.2(3), 2014, pp 676-683.

[14]. Ranganath M S, Vipin, R S Mishra, "Application of ANN for Prediction of Surface Roughness in Turning Process: A Review", International Journal of Advance Research and Innovation, Vol. 3, 2013, pp 229-233.

[15]. Ranganath M S, Vipin, R S Mishra, Neural Network Process Modelling for Turning of Aluminium (6061) using Cemented Carbide Inserts, International Journal of Advance Research and Innovation, Vol. 3,2013, pp 211-219.

[16]. Ranganath M S, Vipin, R S Mishra, "Optimization of surface roughness and material removal rate on conventional dry turning of aluminium (6061)”, International Journal of Advance Research and Innovation, Vol. 2, 2014, pp 62-71. 